\title{
Impaired AMPK-CGRP signaling in the central nervous system contributes to enhanced neuropathic pain in high-fat diet-induced obese rats, with or without nerve injury
}

\author{
XINXIN GUO ${ }^{1,2}$, XUESHU TAO ${ }^{1}$, QING TONG $^{3}$, TIECHENG LI $^{2}$, DAOSONG DONG ${ }^{1}$, \\ BOHAN ZHANG ${ }^{1}$, MENGNAN ZHAO ${ }^{1}$ and TAO SONG ${ }^{1}$ \\ ${ }^{1}$ Department of Pain Medicine, The First Affiliated Hospital of China Medical University, \\ Shenyang, Liaoning 110000; Departments of ${ }^{2}$ Anesthesiology and ${ }^{3}$ Scientific Research, \\ The Third Affiliated Hospital of Jinzhou Medical University, Jinzhou, Liaoning 121000, P.R. China
}

Received November 26, 2018; Accepted May 10, 2019

DOI: $10.3892 / \mathrm{mmr} .2019 .10368$

\begin{abstract}
Obesity is associated with increased sensitivity to pain, including neuropathic pain, but the precise mechanisms are not fully understood. Recent evidence has revealed that AMP-activated protein kinase (AMPK) in the central nervous system (CNS) regulates the neuropeptide calcitonin gene-related peptide (CGRP), a principal neurotransmitter of the class $\mathrm{C}$ nerve fiber, which serves an important role in initiating and maintaining neuropathic pain. AMPK has been demonstrated to be downregulated in the CNS in obesity. The present study hypothesized that obesity may lead to increased sensitivity to neuropathic pain by downregulating AMPK and upregulating CGRP expression levels in the CNS. Sprague-Dawley rats consuming a high-fat diet (HF) for 12 weeks developed obesity; they exhibited significantly decreased levels of phospho (p)-AMPK and increased CGRP expression levels in the spinal cord (SC) and dorsal root ganglion (DRG), respectively, compared with rats consuming a low-fat (LF) diet. HF-fed rats that underwent spared nerve injury (SNI) also exhibited lower p-AMPK and higher CGRP expression levels in the SC and DRG, compared with the corresponding LF-diet rats. The 50\% paw withdrawal threshold (PWT; as measured by Von Frey testing) was significantly lower in HF-fed compared with LF-fed rats, with or without SNI. Through intrathecal treatment, the AMPK activator 5-aminoimidazole-4-carboxamide riboside (AICAR) or the CGRP antagonist CGRP8-37 decreased CGRP expression levels and increased the 50\% PWT; however, the AMPK inhibitor dorsomorphin augmented CGRP expression levels and
\end{abstract}

Correspondence to: Dr Tao Song, Department of Pain Medicine, The First Affiliated Hospital of China Medical University, 155 Nanjing North Street, Heping, Shenyang, Liaoning 110000, P.R. China

E-mail: songt2001@163.com

Key words: obesity, neuropathic pain, AMP-activated protein kinase, calcitonin gene-related peptide further reduced the 50\% PWT in HF-fed rats, but not LF-fed rats, with or without SNI. The results indicated that blocking the AMPK-CGRP pathway may enhance neuropathic pain in HF-induced obesity, with or without nerve injury. Targeting AMPK in the CNS may be a novel strategy for the prevention and treatment of obesity-associated neuropathic pain.

\section{Introduction}

Obesity has reached epidemic proportions worldwide and is associated with insulin resistance, dyslipidemias, type 2 diabetes and neurodegenerative disorders (1). Emerging evidence has revealed a link between obesity and increased sensitivity to pain. For example, obese Zucker rats were found to be more sensitive to thermal stimulation of the tail (2), and diet-induced obese rats exhibited increased paw edema following Freund's complete adjuvant-induced arthritis (3). Also, diet-induced obese rats and obese Zucker rats exhibited augmented peripheral inflammation and inflammatory hyperalgesia in response to an intradermal injection of carrageenan (4-6). In addition, obesity has been associated with enhanced neuropathic pain independent of diabetes (7), though the underlying mechanism remains unclear. There are currently no available pharmacological treatments for the prevention or cure of obesity-associated pain. Therefore, a greater understanding of the precise mechanisms of this pathology, as well as the identification of potential drug targets, is of considerable interest. AMP-activated protein kinase (AMPK) is an important sensor of cellular energy status that serves a key role in cellular energy homeostasis (8). AMPK has been implicated in a number of diseases related to energy metabolism, including obesity. In addition to the regulation of cellular energy metabolism, AMPK may mediate the pain response (as indicated in preclinical pain models) $(9,10)$, and targeting AMPK is now considered to be a novel strategy for the prevention and treatment of pain (11). A recent study demonstrated that the activation of AMPK alleviated trigeminal neuralgia by downregulating the expression level of neuropeptide calcitonin gene-related peptide (CGRP; a principal neurotransmitter of nociceptive sensory $\mathrm{C}$-fibers), and reducing neuroinflammation 
in the spinal trigeminal nucleus (12). The activation of AMPK has also been reported to be impaired in the central nervous system (CNS) of diet-induced obese animals (13).

The present study hypothesized that obesity may result in increased sensitivity to neuropathic pain by downregulating AMPK and upregulating CGRP-associated pathways in the spinal cord (SC) and dorsal root ganglion (DRG). For this purpose, a high-fat diet (HF)-induced obese rat model that mimics human obesity syndrome was utilized, and mechanical allodynia in rats with or without spared nerve injury (SNI) was assessed.

\section{Materials and methods}

Animals. A total of 100 male Sprague-Dawley rats weighing 70 g (4 weeks old; Liaoning Changsheng Biological Center, Shenyang, China) were fed a high-fat diet (HF; fat-containing $45 \%$ kcal; Research Diets, Inc.) to induce obesity as previously described (14). A further 100 male Sprague-Dawley rats were given a low-fat diet (LF; contains $10 \%$ kcal of fat; Research Diets, Inc), and 10 rats were allocated to each group as previously described (14). All animals were housed at a constant temperature of $23-25^{\circ} \mathrm{C}$, with $50 \%$ relative humidity and a 12-h light/dark cycle. The rats had ad libitum access to food and autoclaved water. All experiments were conducted in accordance with the Guiding Principles for Research Involving Animal and Human Beings, and the experimental procedures were approved by the Animal Care and Use Committee of China Medical University (IACUC no. 20180121).

Chemicals and reagents. Phosphorylated (p)-AMPK ${ }^{\text {Thr172 }}$ and total-AMPK antibodies were purchased from Cell Signaling Technology, Inc. 5-Aminoimidazole-4-carboxamide riboside (AICAR),dorsomorphin(DOR) and CGRP8-37 were purchased from APExBIO. The CGRP ELISA kit was purchased from Cusabio Technology LLC (cat. no. CSB-E08211r). All other chemicals were purchased from Sigma-Aldrich; Merck KGaA.

Experimental protocols. To examine neuropathic pain in obese animals, body weight and mechanical allodynia were measured once a week during HF or LF feeding. At 10 and 12 weeks after feeding, 20 rats from each group were sacrificed to collect the SC and DRG for molecular studies. The remaining animals were treated with an intrathecal AMPK activator (AICAR; $0.2 \mu \mathrm{mol} / \mathrm{kg}$ ), AMPK inhibitor (DOR; $0.2 \mu \mathrm{mol} / \mathrm{kg}$ ), CGRP antagonist (CGRP8-37; $20 \mathrm{pmol} / \mathrm{kg}$ ) or the saline vehicle control (VEH) after 12 weeks, for a further 7 days. During treatment, mechanical allodynia was recorded daily, and the SC and DRG were collected at the end of the treatment course.

To investigate neuropathic pain following nerve injury in obese animals, 12 weeks after HF or LF feeding, rats from each group underwent SNI, and mechanical allodynia was measured once a week for 14 days. At the 14-day time-point, these animals were treated with AICAR, DOR or VEH for 7 consecutive days. During treatment, mechanical allodynia was measured daily, and the SC and DRG were collected for further studies following treatment.

Quantitative assessment of allodynia. Mechanical allodynia was assessed using the Von Frey test as previously described (15). Briefly, the rats were placed in a cage with a mesh bottom, and calibrated cilia-Von Frey filaments were applied perpendicularly to the hind paws until a positive response was observed, which included withdrawal and licking or shaking of the paws. The up-down method was used to determine the mechanical force required to induce paw withdrawal in $50 \%$ of animals. A filament that was estimated to be close to the $50 \%$ paw withdrawal threshold (PWT) was first used to stimulate the animal. If the animal showed a positive response, the next lowest-force filament was tested; if the animal did not show a response, the next highest-force filament was tested. A total of 4 readings were acquired and the $50 \%$ threshold was calculated using the following formula: $50 \%$ contraction threshold $=10 \log (\mathrm{x})+\mathrm{k} \delta$; $\mathrm{x}$ was the strength of the last stimulus used; $\mathrm{k}$ was the coefficient of different stimulation modes; and $\delta(\delta=0.224)$ was the average value of the adjacent spacing of each stimulation intensity $(\mathrm{g})$.

SNI animal model. An SNI model that mimics nerve injury-associated human neuropathic pain was induced as previously described (16). Briefly, rats were anesthetized by inhalation of $3 \%$ isoflurane, and the main sciatic nerve and its branches in the hind limb were exposed. The phrenic nerve and the common peroneal nerve were ligated or severed, and small sural nerves were preserved. After $24 \mathrm{~h}$, a significant pain response was triggered in the hind paw and the lateral aspect of the foot.

Lumbar catheterization of the spinal subarachnoid space. For drug delivery to the lumbar subarachnoid space, lumber catheterization was performed as described previously (17). Briefly, the rat was anesthetized by inhalation of $3 \%$ isoflurane. An incision lateral to the midline was made and a guided cannula $(20 \mathrm{G})$ was inserted into the subarachnoid space. The correct localization was indicated by a tail-flicking action and hind limb paralysis after administration of $2 \%$ lidocaine $(10 \mu \mathrm{l})$ through the catheter in wakened animals.

Specimen collection and processing. Rats were euthanized individually in a transparent sealed box; $5 \mathrm{ml} / \mathrm{kg}$ sevoflurane liquid was introduced into the box, and the rat was allowed to inhale the vapor until death ( $2 \mathrm{~min})$. The $\mathrm{L}_{4-6} \mathrm{SC}$ and DRG were isolated from the rat and stored at $-80^{\circ} \mathrm{C}$ for subsequent western blot and ELISA analyses.

Western blotting. The $\mathrm{L}_{4-6} \mathrm{SC}$ and DRG samples were homogenized in $400 \mu \mathrm{l}$ homogenization buffer containing RIPA lysis buffer (cat. no. P0013B; Beyotime Institute of Biotechnology). The samples were incubated at $0^{\circ} \mathrm{C}$ for $30 \mathrm{~min}$, followed by centrifugation at $12,000 \mathrm{x} \mathrm{g}$ at $4^{\circ} \mathrm{C}$ for $10 \mathrm{~min}$. The supernatant was collected and denatured by boiling for $5 \mathrm{~min}$. Total protein was quantified using the BCA method and was separated using $12 \%$ SDS-PAGE gels ( $20 \mu \mathrm{g}$ of protein was loaded per lane), and then transferred to a polyvinylidene fluoride membrane. The membrane was blocked with blocking buffer containing $5 \%$ fat-free milk for $30 \mathrm{~min}$ at room temperature and incubated with the following primary antibodies at $4^{\circ} \mathrm{C}$ overnight: Rabbit anti-p-AMPK ${ }^{\text {Thr172 }}$ (1:1,000; cat. no. 50081s; Cell Signaling Technology, Inc.), rabbit anti-total APMK (1:1,000; cat. no. 5832s; Cell Signaling Technology, 
Inc.) and rabbit anti-GAPDH (1:1,000; cat. no. 5174s; Cell Signaling Technology, Inc.). The membrane was then washed and incubated with a horseradish peroxidase-conjugated goat anti-rabbit secondary antibody (1:10,000; cat. no. zb-2301; ZSGB BIO) for $2 \mathrm{~h}$ at room temperature, prior to developing with ECL reagent (EMD Millipore). The results were acquired and analyzed using a molecular imager (Gel Doc ${ }^{\mathrm{TM}}$ XR; 170-8170) and the associated Quantity One 4.6.5 software (Bio-Rad Laboratories, Inc.). Statistical analysis was performed using the optical density ratio of the target protein bands to that of the GAPDH band, and the expression levels of phosphorylated proteins were normalized according to the following formula: (phosphorylated protein/GAPDH)/(total protein/GAPDH).

ELISA. The concentrations of CGRP in the SC and DRG were measured using an ELISA kit (Thermo Fisher Scientific, Inc.) according to the manufacturer's instructions.

Statistical analysis. All data are presented as the mean \pm standard error of the mean. Statistical analyses were performed using GraphPad Prism 7 (GraphPad Software, Inc.,). Two-way repeated measures ANOVA was conducted on PWT measurements, followed by the Bonferroni correction to identify the significant differences between groups at different time points. ELISA and western blotting results between groups were compared using one- or two-way ANOVA followed by the Bonferroni correction. $\mathrm{P}<0.05$ was considered to indicate a statistically significant difference.

\section{Results}

Mechanical allodynia and CGRP signaling in obesity. Prior to HF feeding, body weight was similar between rats assigned to the HF and LF groups (Fig. 1A). After 6 weeks of feeding, the HF-fed rats had gained significantly more body weight than the LF-fed rats, and this trend continued throughout the 12-week dietary period. After 10 weeks of feeding, the mean body weight in HF-fed rats was approximately double that of the LF-fed rats, indicating that a HF diet resulted in obesity in rats.

Mechanical allodynia was assessed with the Von-Frey test and the 50\% PWT was measured. The 50\% PWT was significantly lower in HF-fed rats compared with LF-fed rats at 10 weeks, and this trend persisted until 12 weeks (Fig. 1B).

The CGRP pathway has been suggested to serve an important role in mediating neuropathic pain $(18,19)$. To examine the possible molecular mechanisms responsible for enhanced mechanical allodynia in HF-fed rats, the expression levels of CGRP in the SC and DRG were measured and were comparable between the groups at 10 weeks, but significantly higher in HF-fed rats than in LF-fed rats at the 12-week point (Fig. 1C).

To further examine whether increased CGRP in the SC and DRG was associated with enhanced mechanical allodynia in HF-fed rats, HF- and LF-fed rats were treated with the intrathecal CGRP antagonist CGRP8-37 or the saline VEH control for 7 consecutive days, after 12 weeks of feeding. As shown in Fig. 1D, the 50\% PWT was significantly lower in HF-fed rats compared with LF-fed rats receiving intrathecal
VEH. Intrathecal CGRP8-37 significantly increased the 50\% PWT in HF-fed rats throughout the 7 days when compared with VEH treatment. Intrathecal CGRP8-37 did not alter the $50 \%$ PWT in LF-fed rats.

Effects of modulating the AMPK pathway on mechanical allodynia in obesity. Activation of AMPK has been shown to mediate neuropathic pain via the CGRP pathway (11); therefore, the role of the AMPK pathway in regulating neuropathic pain in obesity was examined. As presented in Fig. 2, intrathecal treatment with AICAR for 7 days significantly increased the 50\% PWT in HF-fed rats compared with VEH treatment (Fig. 2A). By contrast, intrathecal treatment with the AMPK inhibitor DOR for 7 days further decreased the 50\% PWT in HF-fed rats compared with VEH treatment (Fig. 2B). Neither AICAR nor DOR had any effect on the 50\% PWT in LF-fed rats.

The expression of $\mathrm{p}-\mathrm{AMPK}^{\mathrm{Thr} 172}$ in the SC was significantly lower in HF-fed rats at both 10 and 12 weeks, compared with that in LF-fed rats (Fig. 2C). The level of p-AMPK ${ }^{\text {Thr172 }}$ in the SC of HF-fed rats was increased by intrathecal treatment with AICAR, decreased by DOR treatment, and unchanged by CGRP8-37 treatment (Fig. 2D). Additionally, the levels of CGPR in the SC of HF-fed rats were decreased by AICAR and CGRP8-37 treatment, but increased by DOR treatment (Fig. 2E). None of the treatments had any notable effect on p-AMPK ${ }^{\text {Thr172 }}$ or CGPR expression levels in LF-fed rats.

Mechanical allodynia and the AMPK-CGRP pathway in obesity after SNI. To investigate neuropathic pain following nerve injury in obese animals, SNI was induced after 12 weeks of HF or LF feeding, and mechanical allodynia was measured for 14 consecutive days. At 2 weeks after SNI, rats were treated with intrathecal AICAR, DOR, CGRP8-37 or $\mathrm{VEH}$, and mechanical allodynia was measured for another 7 consecutive days. As shown in Fig. 3, HF-fed rats exhibited a significantly lower $50 \%$ PWT than LF-fed rats at all time points following SNI (Fig. 3A). Intrathecal treatment with AICAR (Fig. 3B) or CGRP8-37 (Fig. 3C) increased the 50\% PWT, whereas DOR (Fig. 3D) further reduced the 50\% PWT in HF-fed rats compared with LH-fed rats.

The expression levels of CGRP in the SC (Fig. 4A) and DRG (Fig. 4B) were significantly higher in HF-fed rats than in LF-fed rats following SNI. Intrathecal treatment with AICAR or CGRP8-37 reduced the levels of CGRP expression in the SC and DRG in both study groups. By contrast, intrathecal treatment with DOR augmented the expression levels of CGRP in the SC and DRG of HF-fed rats, compared with those in LF-fed rats. Moreover, p-AMPK ${ }^{\text {Thr172 }}$ (Fig. 4C) in the SC was lower in HF-fed rats compared with LF-fed rats after SNI. Intrathecal treatment with AICAR or CGRP8-37 upregulated p-AMPK ${ }^{\mathrm{Thr} 172}$ in SC, whereas intrathecal treatment with DOR decreased the expression of $\mathrm{p}-\mathrm{AMPK}^{\mathrm{Thr}}{ }^{72}$ in the $\mathrm{SC}$ of HF-fed rats compared with that in LF-fed rats after SNI. As the present sudy focused on the effect of neuropathic pain in rats fed a HF diet, there was no concern about some changes in the rats fed a LF diet. A significant decrease in the expression of p-AMPK ${ }^{\text {Thr172 }}$ was not observed in the LF group after DOR. It is hypothesized that the SNI model itself may have an effect on the expression of $\mathrm{p}-\mathrm{AMPK}^{\mathrm{Thr} 172}$ 

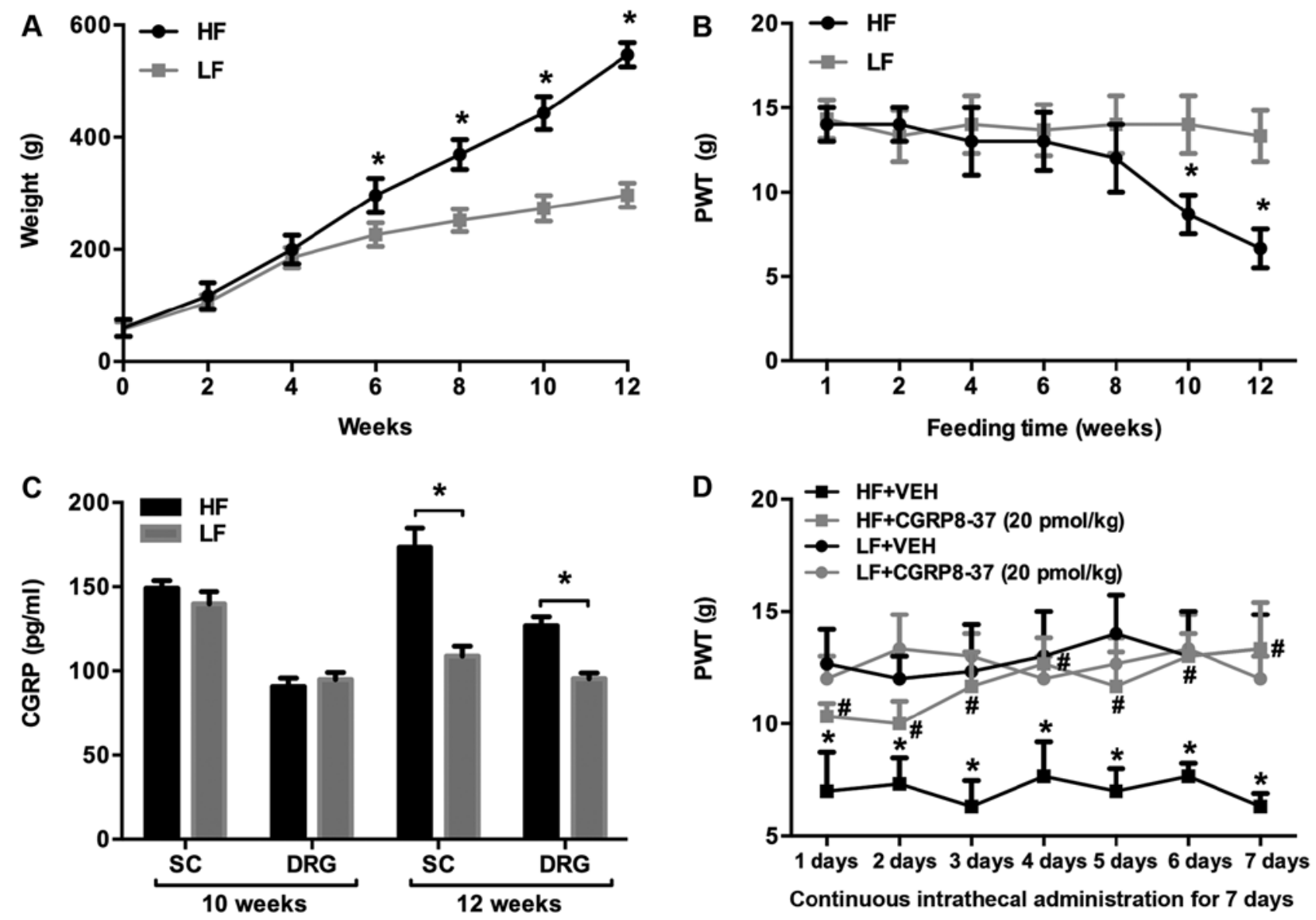

Figure 1. PWT, body weight and CGRP expression in rats fed with HF or LF diets for 12 weeks. Alterations in (A) body weight, (B) $50 \%$ PWT (as measured by the Von Frey test) and (C) expression levels of CGRP in the SC and DRG. "P<0.05 vs. respective LF group. (D) Effects of intrathecal treatment with a CGRP antagonist (CGRP8-37) on 50\% PWT in rats fed an HF or LF diet. " $\mathrm{P}<0.05$ vs. LF+VEH; "P<0.05 vs. HF+VEH. n=10 per group. PWT, paw withdrawal threshold; CGRP, calcitonin gene-related peptide; SC, spinal cord; DRG, dorsal root ganglion; HF, high fat; LF, low fat; VEH, vehicle.

\section{Discussion}

The primary findings of the present study were: i) HF-induced obese rats with or without nerve injury exhibited enhanced mechanical pain sensitivity, which was inhibited by the AMPK activator AICAR or the CGRP antagonist CGRP8-37, and exaggerated by the AMPK inhibitor DOR; ii) HF-induced obese rats with or without nerve injury showed decreased levels of $\mathrm{p}$-AMPK ${ }^{\mathrm{Thr} 172}$ and higher expression levels of CGRP in the SC and DRG; iii) p-AMPK ${ }^{\text {Thr172 }}$ in the SC and DRG of $\mathrm{HF}$-induced obese rats was upregulated by AICAR, which also reduced the expression levels of CGRP; and iv) p-AMPK ${ }^{\text {Thr172 }}$ in the SC and DRG of HF-induced obese rats was downregulated by DOR, which also augmented the expression levels of CGRP. Collectively, these data suggested that the enhanced neuropathic pain in $\mathrm{HF}$-induced obese rats with or without nerve injury was due to decreased AMPK activity in the SC and DRG, which resulted in increased CGRP expression levels.

Neuropathic pain is defined as pain resulting from injury or disease affecting the peripheral nervous system or CNS. Injury to the peripheral nervous system due to trauma, metabolic disease or exposure to drugs is the fundamental etiology of neuropathic pain (20). The common symptomology of neuropathic pain includes exquisite hypersensitivity to mechanical stimulation (20). A recent study demonstrated that HF-induced obese animals exhibited increased neuropathic pain (21), but the mechanisms are not fully understood. Additionally, it is unclear whether neuropathic pain is enhanced in obesity following nerve injury. In the present study, it was discovered that HF-induced obese rats possessed enhanced mechanical pain sensitivity compared with LF-fed control rats, which is consistent with a previous study (21). The present study also extended previous research by demonstrating that mechanical pain sensitivity remained higher in HF-induced obese rats than in LF-fed control rats after nerve injury (22).

Emerging evidence has indicated that the AMPK pathway in the SC and DRG serves a prominent role in the mediation of neuropathic pain (9). The activation of AMPK negatively regulates activity-dependent protein synthesis and other signaling pathways that drive neuronal plasticity in neuropathic pain, resulting in reduced nociceptor excitability (23). Systemic activation of AMPK with either metformin or A769662 in rodents, following peripheral nerve injury, attenuates mechanical hypersensitivity $(24,25)$. Additionally, activation of AMPK in the SC in a rat model of neuropathic pain reduced mechanical hypersensitivity by inhibiting the expression of proinflammatory cytokines, and upregulating that of the glutamate transporter after nerve injury (26). In vitro experiments using patch-clamp electrophysiology on DRG neurons demonstrate that the activation of AMPK 


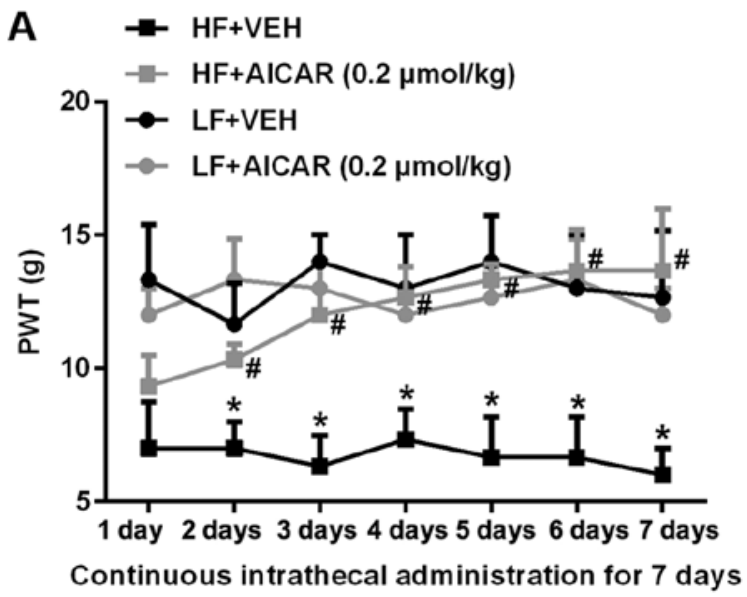

C
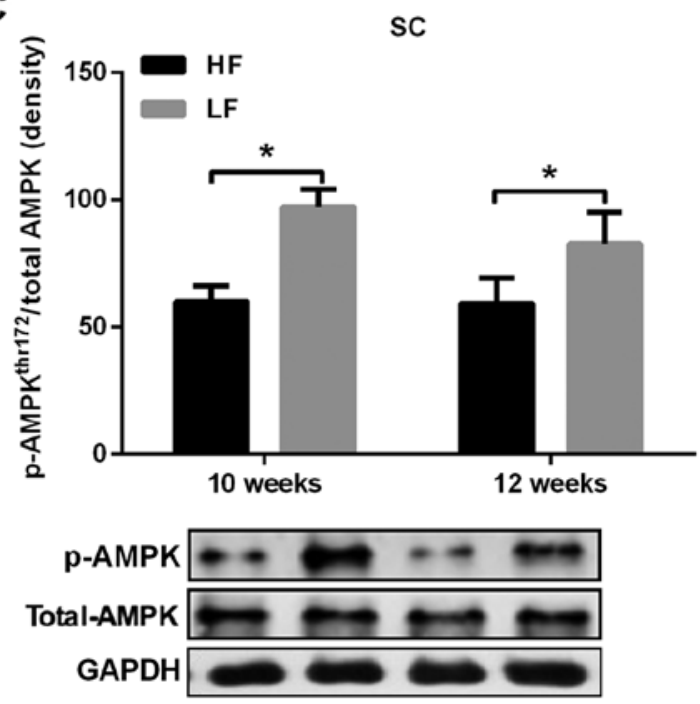

E

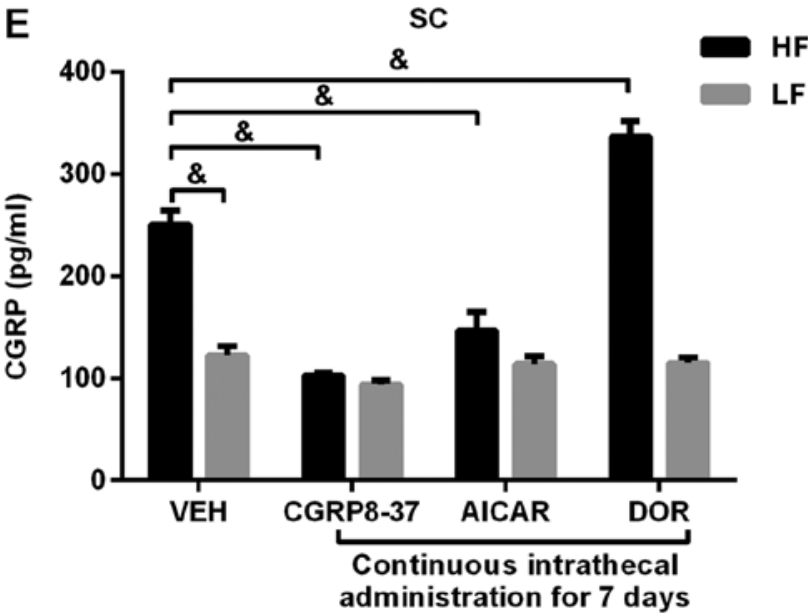

B

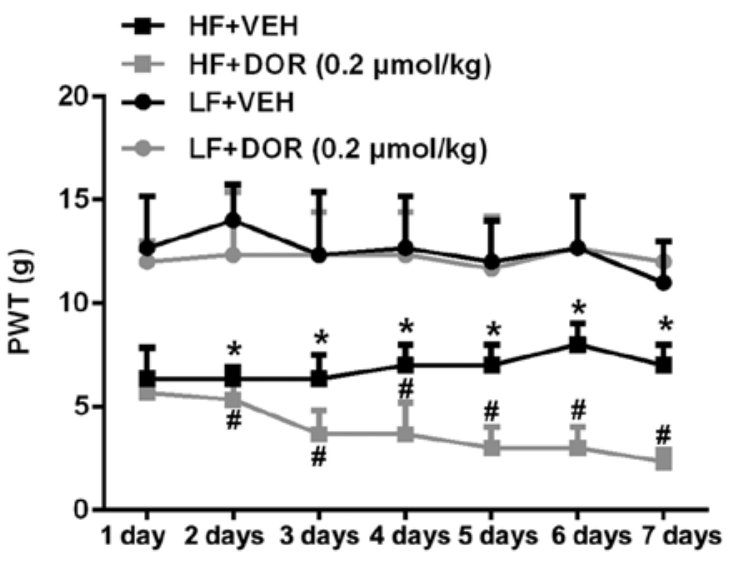

Continuous intrathecal administration for 7 days

D

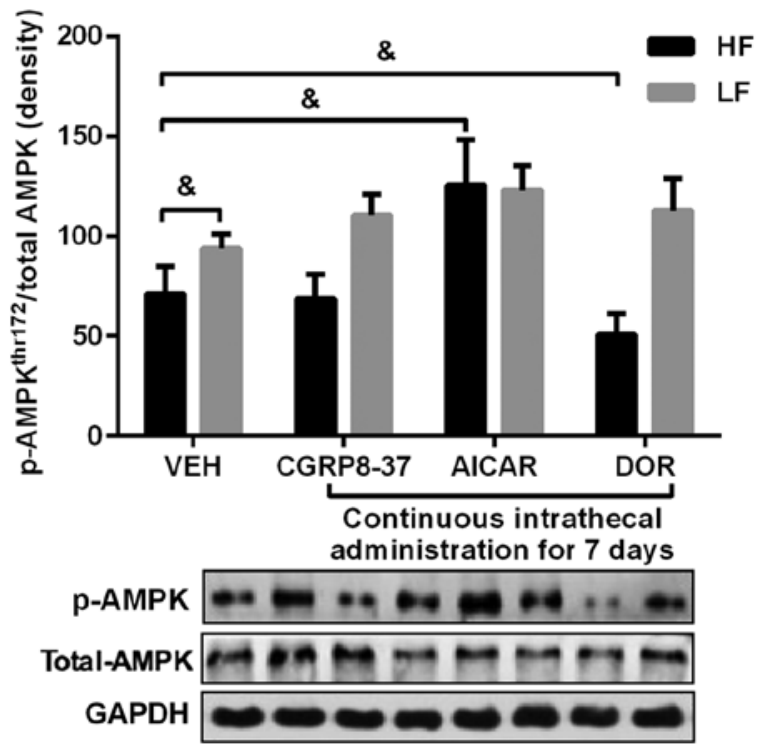

Figure 2. PWT, expression of p-AMPK and CGRP in rats given an AMPK agonist or inhibitor. (A) $50 \%$ PWT in HF-fed rats and LF-fed rats treated with the intrathecal AMPK activator AICAR. "P<0.05 vs. respective LF group; ${ }^{~} \mathrm{P}<0.05$ vs. respective HF+VEH group. (B) $50 \%$ PWT in HF-fed rats and LF-fed rats treated with the intrathecal AMPK inhibitor DOR. ${ }^{*} \mathrm{P}<0.05$ vs. respective $\mathrm{LF}$ group; ${ }^{\mathrm{B}} \mathrm{P}<0.05$ vs. respective HF+DOR group. (C) Expression levels of p-AMPK ${ }^{\text {Thr172 }}$ in the SC at 10 and 12 weeks after HF or LF feeding. " $\mathrm{P}<0.05$. (D) Effect of intrathecal AICAR, DOR and CGRP8-37 on p-AMPK ${ }^{\text {Thr172 }}$ in HF- and LF-fed rats. (E) Effects of intrathecal AICAR, DOR and CGRP8-37 on expression levels of CGRP in the SC of HF- and LF-fed rats. ${ }^{\text {\& }} \mathrm{P}<0.05$. $\mathrm{n}=10 \mathrm{per}$ group. PWT, paw withdrawal threshold; HF, high fat; LF, low fat; AMPK, AMP-activated protein kinase; AICAR, 5-aminoimidazole-4-carboxamide riboside; DOR, dorsomorphin; p-AMPK, phosphorylated-AMPK; SC, spinal cord; CGRP, calcitonin gene-related peptide; VEH, vehicle.

with metformin or A769662 reduces the excitability of DRG neurons; these neurons are widely believed to induce ectopic activity causing an ongoing burning pain, a prominent feature of neuropathic pain following injury (24). In the present study, p-AMPK ${ }^{\text {Thr172 }}$ levels in the SC and DRG were significantly lower in HF-induced obese rats compared with LF-fed control 
A

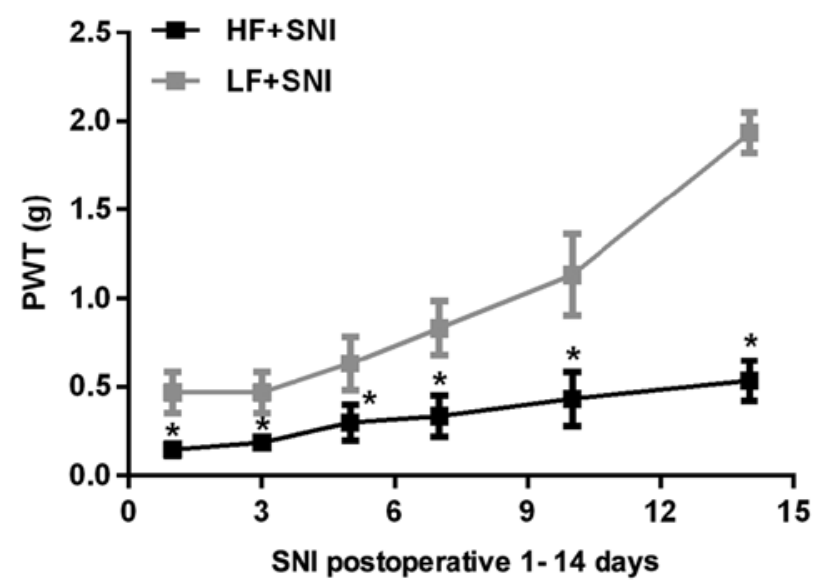

C

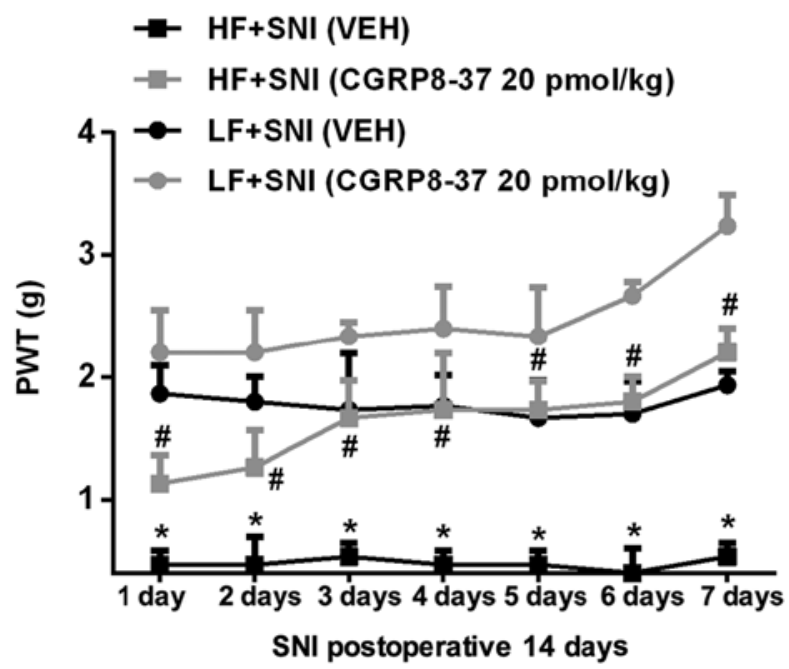

B

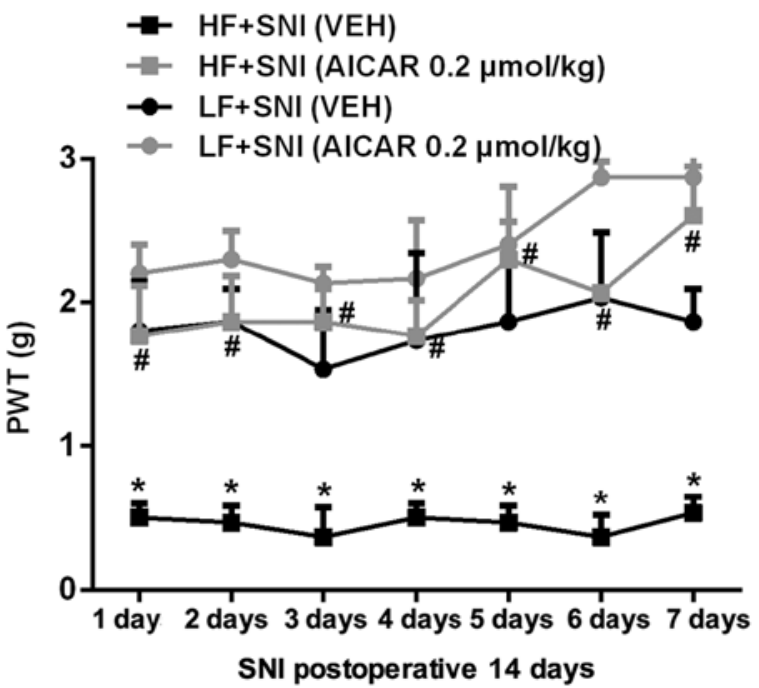

D

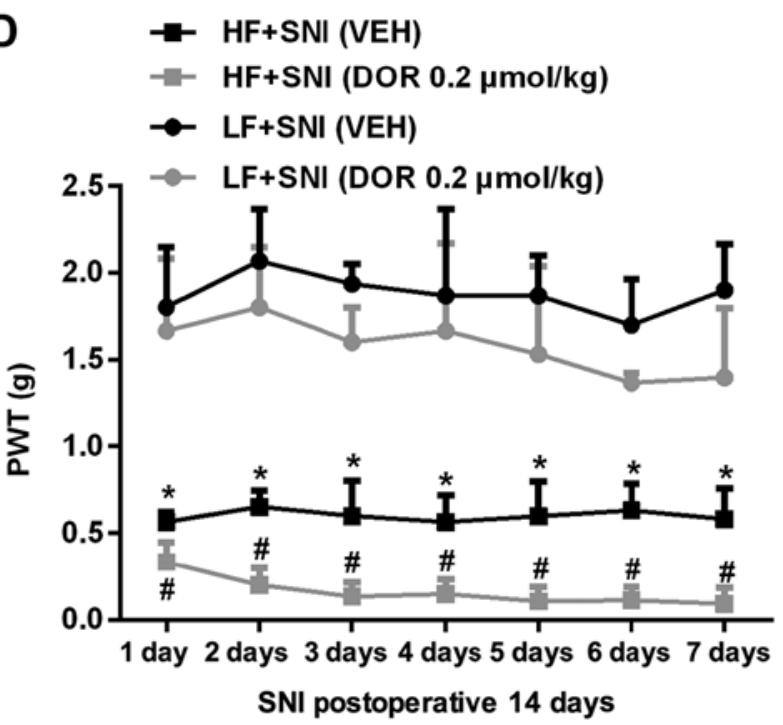

Figure 3. PWT 14 days after SNI and following administration of an AMPK agonist or inhibitor for 7 days. (A) 50\% PWT in HF- and LF-fed rats following SNI. "P<0.05 vs. respective LF+SNI group. (B) Effect of intrathecal AICAR on 50\% PWT in HF- and LF-fed rats with SNI. (C) Effect of intrathecal CGRP8-37 on 50\% PWT in HF- and LF-fed rats with SNI. (D) Effect of intrathecal DOR on 50\% PWT in HF- and LF-fed rats with SNI. "P<0.05 vs. respective LF+SNI (VEH) group; ${ }^{~} \mathrm{P}<0.05$ vs. respective $\mathrm{HF}+\mathrm{SNI}$ (VEH). $\mathrm{n}=10$ per group. PWT, paw withdrawal threshold; HF, high fat; LF, low fat; SNI, spared nerve injury; AICAR, 5-aminoimidazole-4-carboxamide riboside; DOR, dorsomorphin; VEH, vehicle.

rats after 10 weeks of HF feeding, indicating that AMPK signaling was impaired in HF-induced obesity. This finding is consistent with a previous study illustrating impaired AMPK-signaling in the CNS of HF-induced obese rats (13). To understand whether impaired AMPK-signaling in the SC and DRG accounts for enhanced mechanical pain sensitivity in obesity, animals were treated with the intrathecal AMPK activator AICAR or AMPK inhibitor DOR. This revealed that AICAR increased AMPK activity in the SC and DRG of HF-induced obese rats with or without nerve injury, leading to decreased mechanical pain sensitivity. By contrast, intrathecal DOR further reduced AMPK activity in the SC and DRG in $\mathrm{HF}$-induced obese rats (with or without nerve injury), resulting in exaggerated mechanical pain sensitivity. These data clearly demonstrated that impaired AMPK activity in the SC and DRG may be a primary contributor to enhanced neuropathic pain in HF-induced obesity.
CGRP is a neuropeptide that is primarily generated in the central and peripheral nervous systems, particularly in the SC, DRG and trigeminal ganglion (27). CGRP in the SC serves an important role in chronic pain by facilitating the introduction of synaptic pain information via protein kinase $\mathrm{A}$ and protein kinase $\mathrm{C}$ second messenger pathways, and participating in the generation and maintenance of allodynia and hyperpathia (27-30). CGRP expression levels were increased in animals after peripheral nerve injury, and serve an important role in generating and maintaining pain behavior $(27,31)$. In the present study, the 50\% PWT in HF-fed rats was significantly reduced compared with that of LF-fed rats, at both 10 and 12 weeks of feeding. Additionally, the expression levels of CGRP in the SC and DRG of HF-fed rats were significantly higher than in LF-fed rats at 12 weeks. However, there was no significant difference between the two groups at 10 weeks. Furthermore, the $50 \%$ PWT of the HF group decreased 

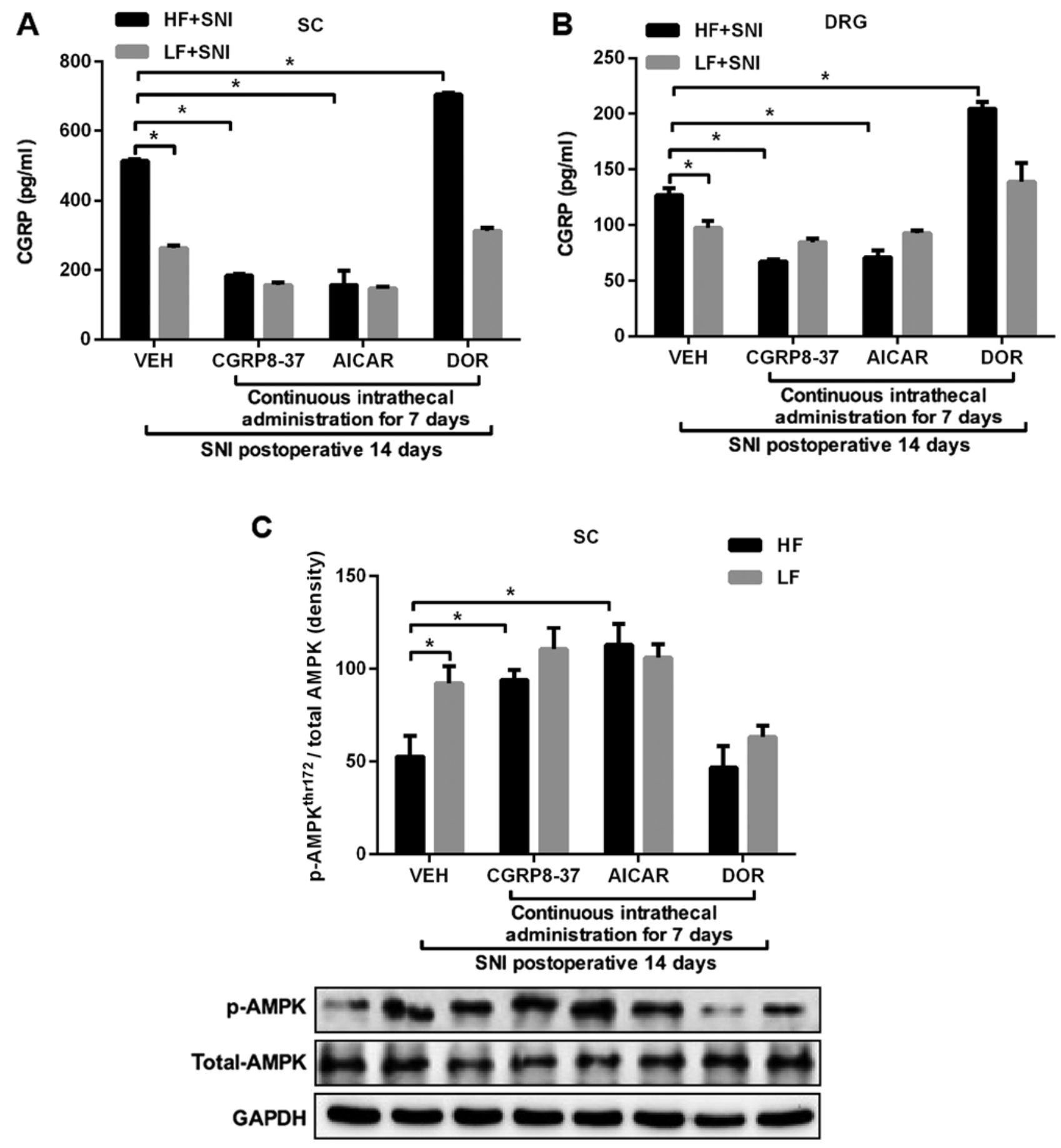

Figure 4. Expression of p-AMPK and CGRP 14 days after SNI and following administration of an AMPK agonist or inhibitor for 7 days. Effects of intrathecal AICAR, DOR and CGRP8-37 treatment on the expression levels of CGRP in the (A) SC and (B) DRG of HF- and LF-fed rats with SNI. (C) Effect of intrathecal AI, DOR or CGRP8-37 on p-AMPK ${ }^{\text {Thr172 }}$ in the SC in HF- and LF-fed rats with SNI. ${ }^{\mathrm{P}}<0.05$. $\mathrm{n}=10$ per group. AICAR, 5-aminoimidazole-4-carboxamide riboside; DOR, dorsomorphin; CGRP, calcitonin gene-related peptide; SC, spinal cord; DRG, dorsal root ganglion; HF, high fat; LF, low fat; SNI, spared nerve injury; p-AMPK, phosphorylated-AMPK; VEH, vehicle.

significantly at 10 weeks, which may have been associated with oxidative stress. Previously, increased levels of oxidative stress biomarkers such as malondialdehyde (MDA), SOD and reactive oxygen species (ROS). have been detected in the plasma of obese patients $(31,32)$. Animal models have also revealed that weight loss, dietary restriction and exercise can also decrease the expression levels of these biomarkers (33). There is increasing evidence that the nervous system undergoes oxidative stress reactions following noxious stimulation and inflammation, and that the production of oxidation products increases; this results in damage to nerve tissue, which is an important contributor to neuropathic pain $(34,35)$. Zhao et al (36) found that MDA concentrations increased, and that superoxide dismutase activity was decreased in a neuropathic pain rat model. The present study indicated that AMPK phosphorylation was also significantly reduced in the HF group at 10 weeks; this suggests that AMPK may have an important regulatory effect on intracellular oxidative stress, 
which may subsequently inhibit the generation of $\operatorname{ROS}(37,38)$. Therefore, it was hypothesized that the $50 \%$ PWT reduction may be associated with reduced AMPK phosphorylation, resulting in increased levels of oxidative stress; this will be the focus of future investigations.

Inhibiting CGRP expression in the SC has been proven to significantly ameliorate pain behavior after peripheral nerve injury $(27,29)$. A recent study revealed that AMPK mediates neuropathic pain by regulating CGRP (12). Consistent with previous studies $(27,31)$, the present study observed a significant increase in CGRP expression levels in the SC and DRG of HF-induced obese rats (with or without nerve injury), and treatment with CGRP8-37 prevented an increase in mechanical pain sensitivity to a significant degree; this indicates that increased HF-induced obesity may result in increased levels of CGRP expression in the SC and DRG, contributing to enhanced neuropathic pain. Moreover, the levels of CGRP expression in the SC and DRG of HF-induced obese rats were decreased by AICAR, but further increased by the AMPK inhibitor DOR, indicating that AMPK in the SC and DRG mediates neuropathic pain in HF-induced obesity via the CGRP pathway.

The present study revealed that the administration of an AMPK agonist (AICAR) or CGRP inhibitor (CGRP8-37) significantly increased the 50\% PWT compared with VEH administration in HF-fed rats, but neither AICAR nor CGRP8-37 had this effect in LF-rats. Obesity is associated with multiple chronic conditions that lead to pain, such as osteoarthritis, peripheral vascular disease, lower back pain and neuropathic pain (39-42). In addition, overweight patients appear to experience more severe paroxysmal pain, and their neuropathic-negative symptoms tend to be aggravated (43). A large number of studies have revealed that neuropathic pain is associated with increased expression levels of proinflammatory factors (including IL-1 $\beta$, IL-6 and IL-8); adipose tissue is an endocrine organ, generating various cytokines such as leptin, adiponectin, TNF- $\alpha$, interleukins (IL-1 $\beta$, IL-6, IL-8, IL-10) and other biologically active factors involved in metabolism and immunity $(44,45)$. Studies have shown that serum expression levels of TNF- $\alpha$ and IL-6 are significantly decreased in obese patients after strict dietary intake (46). Therefore, it was hypothesized that AMPK reduction, CGRP enhancement and increased neuropathic pain may be reversed following weight loss in HF-induced obese rats, though further experiments are needed to verify this claim.

In conclusion, the present study demonstrated that AMPK activity was reduced in the SC and DRG of HF-induced obese rats. Reduced AMPK activity resulted in increased CGRP production in the SC and DRG, contributing to enhanced neuropathic pain in HF-induced obese rats, with or without nerve injury. These results suggested that obesity may decrease AMPK activity in the CNS, indicating that AMPK may be a novel therapeutic target for obesity-related neuropathic pain.

\section{Acknowledgements}

Not applicable.

\section{Funding}

The present study was supported by the Medical Health Joint Fund (grant no. 20180530063) and the Guidance Project Fund (grant no. 20180550175) from the Liaoning Natural Science Foundation of China.

\section{Availability of data and materials}

The datasets generated and analyzed during the present study are available from the corresponding author on reasonable request.

\section{Authors' contributions}

XG performed the majority of the experiments, designed the study and acquired data, was involved in the analysis and interpretation of data, participated in drafting the manuscript, in critical revision of important content and gave final approval of the version to be released. XT, TL and QT were involved in drafting the manuscript and acquisition of data. DD, XT, $\mathrm{BZ}$ and $\mathrm{MZ}$ contributed to data interpretation and analysis. TS made a substantial contribution to the experimental design, drafting and critically revising the manuscript. All of the authors have read and approved the final manuscript.

\section{Ethics approval and consent to participate}

All experiments were conducted in accordance with the Guiding Principles for Research Involving Animal and Human Beings, and the experimental procedures were approved by the Animal Care and Use Committee of China Medical University (IACUC no. 20180121).

\section{Patient consent for publication}

Not applicable.

\section{Competing interests}

The authors declare that they have no competing interests.

\section{References}

1. Schwartz MW, Seeley RJ, Zeltser LM, Drewnowski A, Ravussin E, Redman LM and Leibel RL: Obesity Pathogenesis: An endocrine society scientific statement. Endocr Rev 38: 267-296, 2017.

2. Roane DS and Porter JR: Nociception and opioid-induced analgesia in lean (Fa/-) and obese (fa/fa) Zucker rats. Physiol Behav 38: 215-218, 1986.

3. Croci T and Zarini E: Effect of the cannabinoid CB1 receptor antagonist rimonabant on nociceptive responses and adjuvant-induced arthritis in obese and lean rats. Br J Pharmacol 150: 559-566, 2007.

4. Iannitti T, Graham A and Dolan S: Increased central and peripheral inflammation and inflammatory hyperalgesia in Zucker rat model of leptin receptor deficiency and genetic obesity. Exp Physiol 97: 1236-1245, 2012.

5. Wang J,Zhang Q,ZhaoL,LiD, Fu Z and Liang L: Down-regulation of PPAR $\alpha$ in the spinal cord contributes to augmented peripheral inflammation and inflammatory hyperalgesia in diet-induced obese rats. Neuroscience 278: 165-178, 2014.

6. Zhang Y, Song C, Li H, Hou J and Li D: Ursolic acid prevents augmented peripheral inflammation and inflammatory hyperalgesia in high-fat diet-induced obese rats by restoring downregulated spinal PPAR $\alpha$. Mol Med Rep 13: 5309-5316, 2016.

7. Hozumi J, Sumitani M, Matsubayashi Y, Abe H, Oshima Y, Chikuda H, Takeshita K and Yamada Y: Relationship between neuropathic pain and obesity. Pain Res Manag 2016: 2487924, 2016. 
8. Kim M and Tian R: Targeting AMPK for cardiac protection: Opportunities and challenges. J Mol Cell Cardiol 51: 548-553, 2011.

9. Kialka M, Doroszewska K, Janeczko M and Milewicz T: Metformin-new potential medicine in pain treatment? Przegl Lek 74: 81-83, 2017 (In Polish).

10. Price TJ, Das V and Dussor G: Adenosine Monophosphateactivated Protein Kinase (AMPK) Activators For the Prevention, Treatment and Potential Reversal of Pathological Pain. Curr Drug Targets 17: 908-920, 2016.

11. Asiedu MN, Dussor G and Price TJ: Targeting AMPK for the alleviation of pathological pain. Exp Suppl 107: 257-285, 2016.

12. Yang YJ, Hu L, Xia YP, Jiang CY, Miao C, Yang CQ, Yuan M and Wang L: Resveratrol suppresses glial activation and alleviates trigeminal neuralgia via activation of AMPK J Neuroinflammation 13: 84, 2016

13. Fei W, Tian DR, Tso P and Han JS: Diet-induced obese rats exhibit impaired LKB1-AMPK signaling in hypothalamus and adipose tissue. Peptides 35: 23-30, 2012.

14. Song T, Lv LY, Xu J, Tian ZY, Cui WY, Wang QS, Qu G and Shi XM: Diet-induced obesity suppresses sevoflurane preconditioning against myocardial ischemia-reperfusion injury: Role of AMP-activated protein kinase pathway. Exp Biol Med (Maywood) 236: 1427-1436, 2011.

15. Chaplan SR, Bach FW, Pogrel JW, Chung JM and Yaksh TL: Quantitative assessment of tactile allodynia in the rat paw. J Neurosci Methods 53: 55-63, 1994.

16. Decosterd I and Woolf CJ: Spared nerve injury: An animal model of persistent peripheral neuropathic pain. Pain 87: 149-158, 2000

17. Storkson RV, Kjorsvik A, Tjolsen A and Hole K: Lumbar catheterization of the spinal subarachnoid space in the rat. J Neurosci Methods 65: 167-172, 1996.

18. Kanai Y, Nakazato E, Fujiuchi A, Hara T and Imai A: Involvement of an increased spinal TRPV1 sensitization through its up-regulation in mechanical allodynia of CCI rats. Neuropharmacology 49: 977-84, 2005.

19. Rossi HL, Luu AK, DeVilbiss JL and Recober A: Obesity increases nociceptive activation of the trigeminal system. Eur J Pain 17: 649-653, 2013

20. Baron R, Binder A and Wasner G: Neuropathic pain: Diagnosis, pathophysiological mechanisms, and treatment. Lancet Neurol 9: 807-819, 2010.

21. Gavini CK, Bookout AL, Bonomo R, Gautron L, Lee S and Mansuy-Aubert V: Liver X receptors protect dorsal root ganglia from obesity-induced endoplasmic reticulum stress and mechanical allodynia. Cell Rep 25: 271-277 e4, 2018.

22. Price TJ and Dussor G: AMPK: An emerging target for modification of injury-induced pain plasticity. Neurosci Lett $557 \mathrm{Pt}$ A: 9-18, 2013.

23. Melemedjian OK, Asiedu MN, Tillu DV, Sanoja R, Yan J, Lark A, Khoutorsky A, Johnson J, Peebles KA, Lepow T, et al: Targeting adenosine monophosphate-activated protein kinase (AMPK) in preclinical models reveals a potential mechanism for the treatment of neuropathic pain. Mol Pain 7: 70, 2011.

24. Melemedjian OK, Tillu DV, Asiedu MN, Mandell EK, Moy JK, Blute VM, Taylor CJ, Ghosh S and Price TJ: BDNF regulates atypical PKC at spinal synapses to initiate and maintain a centralized chronic pain state. Mol Pain 9: 12, 2013.

25. Maixner DW, Yan X, Gao M, Yadav R and Weng HR: Adenosine monophosphate-activated protein kinase regulates interleukin-1beta expression and glial glutamate transporter function in rodents with neuropathic pain. Anesthesiology 122 $1401-1413,2015$

26. Ren H, Jin H, Jia Z, Ji N and Luo F: Pulsed Radiofrequency Applied to the Sciatic Nerve Improves Neuropathic Pain by Down-regulating The Expression of Calcitonin Gene-related Peptide in the Dorsal Root Ganglion. Int J Med Sci 15: 153-160, 2018

27. Pezet S and McMahon SB: Neurotrophins: mediators and modulators of pain. Annu Rev Neurosci 29: 507-538, 2006

28. Lee SE and Kim JH: Involvement of substance P and calcitonin gene-related peptide in development and maintenance of neuropathic pain from spinal nerve injury model of rat. Neurosci Res 58: 245-249, 2007.

29. Sun RQ, Lawand NB and Willis WD: The role of calcitonin gene-related peptide (CGRP) in the generation and maintenance of mechanical allodynia and hyperalgesia in rats after intradermal injection of capsaicin. Pain 104: 201-208, 2003.
30. Hu P, Bembrick AL, Keay KA and McLachlan EM: Immune cell involvement in dorsal root ganglia and spinal cord after chronic constriction or transection of the rat sciatic nerve. Brain Behav Immun 21: 599-616, 2007.

31. Vincent HK and Taylor AG: Biomarkers and potential mechanisms of obesity-induced oxidant stress in humans. Int J Obes 30 400-418, 2006.

32. Natoli R, Fernando N, Dahlenburg T, Jiao H, Aggio-Bruce R, Barnett NL, Chao de la Barca JM, Tcherkez G, Reynier P, Fang J, Chu-Tan JA, et al: Obesity-induced metabolic disturbance drives oxidative stress and complement activation in the retinal environment. Mol Vis 24: 201-217, 2018.

33. Dandona P, Mohanty P, Ghanim H, Aljada A, Browne R, Hamouda W, Prabhala A, Afzal A and Garg R: The suppressive effect of dietary restriction and weight loss in the obese on the generation of reactive oxygen species by leukocytes, lipid peroxidation, and protein carbonylation. J Clin Endocrinol Metab 86: 355-362, 2001.

34. Kiasalari Z, Rahmani T, Mahmoudi N, Baluchnejadmojarad T and Roghani M: Diosgenin ameliorates development of neuropathic pain in diabetic rats: Involvement of oxidative stress and inflammation. Biomed Pharmacothe 86: 654-661, 2017.

35. Siotto M, Aprile I, Simonelli I, Pazzaglia C, Ventriglia M, Santoro M and Padua L: An exploratory study of BDNF and oxidative stress marker alterations in subacute and chronic stroke patients affected by neuropathic pain. J Neural Transm (Vienna) 124: 1557-1566, 2017.

36. Zhao B, Pan Y, Wang Z, Tan Y and Song X: Intrathecal administration of tempol reduces chronic constriction injury-induced neuropathic pain in rats by increasing SOD activity and inhibiting NGF expression. Cell Mol Neurobiol 36: 893-906, 2015.

37. Lee SG, Wu HM, Lee CG, Oh CS, Chung SW and Kim SG: Binge alcohol intake after hypergravity stress sustainably decreases AMPK and transcription factors necessary for hepatocyte survival. Alcohol Clin Exp Res 41: 76-86, 2017.

38. Shi L, Zhang T, Zhou Y, Zeng X, Ran L, Zhang Q, Zhu J and Mi M: Dihydromyricetin improves skeletal muscle insulin sensitivity by inducing autophagy via the AMPK-PGC-1 $\alpha$-Sirt3 signaling pathway. Endocrine 50: 378-389, 2015.

39. Reyes C, Leyland KM, Peat G, Cooper C, Arden NK and Prieto-Alhambra D: Ass-ociation between overweight and obesity and risk of clinically diagnosed knee, hip, and hand osteoarthritis: A population-based cohort study. Arthritis Rheumatol 68: 1869-1875, 2016.

40. Fischer K, PrzepieraBedzak H, Sawicki M, Walecka A, Brzosko I and Brzosko M: Serum Interleukin-23 in polish patients with systemic lupus erythematosus: Association with lupus nephritis, obesity, and peripheral vascular disease. Mediators Inflamm 2017: 9401432, 2017.

41. Dario AB, Ferreira ML, Refshauge KM, Lima TS, Ordoñana JR and Ferreira PH: The relationship between obesity, low back pain, and lumbar disc degeneration when genetics and the environment are considered: A systematic review of twin studies. Spine J 15: 1106-1117, 2015.

42. Gallagher HC, Gallagher RM, Butler M, Buggy DJ and Henman MC: Venlafaxine for neuropathic pain in adults. Cochrane Database Syst Rev 8: CD011091, 2015.

43. Hozumi J, Sumitani M, Matsubayashi Y, Abe H, Oshima Y, Chikuda $\mathrm{H}$ and Takeshita $\mathrm{K}$ and Yamada Y: Relationship between neuropathic pain and obesity. Pain Res Manag 2016: 2487924, 2016

44. Bruun JM, Stallknecht B, Helge JW and Richelsen B: Interleukin-18 in plasma and adipose tissue: Effects of obesity, insulin resistance and weght loss. EurJ Endocrinl 157: 465-471, 2007.

45. Wisse BE: The inflammatory syndrome: The role of adipose tissue cytokines in metabolic disorders linked to obesity. J Am Soc Nephrol 15: 2792-2800, 2004.

46. Barisione M, Carlini F, Gradaschi R, Camerini G and Adami GF: Body weight at developmental age in siblings born to mothers before and after surgically induced weight loss. Surg Obes Relat Dis 8: 387-391, 2012.

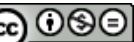

This work is licensed under a Creative Commons Attribution-NonCommercial-NoDerivatives 4.0 International (CC BY-NC-ND 4.0) License. 